\title{
A SIMPLE PROOF OF THE EXTENSION THEOREM OF SEQUENCES OF DIVIDED POWERS IN CHARACTERISTIC $p$
}

\author{
MITSUHIRO TAKEUCHI ${ }^{1}$
}

ABSTRACT. Using the idea of relative Hopf modules, a short proof of the extension theorem of sequences of divided powers in irreducible cocommutative Hopf algebras over a field of characteristic $p>0$ is presented.

Let $k$ be a field of characteristic $p>0$. Let $H$ be an irreducible cocommutative Hopf algebra over $\kappa$. The $V$-map for $H[1,(4.1)]$

$$
V: H \rightarrow k^{1 / p} \otimes H
$$

is a $\frac{1}{p}$-linear Hopf algebra map [1, p. 279] with kernel $H L$ where $L=P(H)$, the primitives in $H$ [4, Theorem 1]. We define a descending set of Hopf subalgebras $\left\{V^{n}(H)\right\}_{n>0}$ inductively as follows: $V^{0}(H)=H, V^{n}(H)=V\left(V^{n-1}(H)\right) \cap H$. $\left(V^{1}(H)=V(H) \cap H\right.$ is different from $V(H)$.) Since $V(H)$ is a $k^{1 / p_{-}}$-Hopf subalgebra of $k^{1 / p} \otimes H$, it is easy to check that each $V^{n}(H)$ is a $k$-Hopf subalgebra of $H$. An element $x \in H$ has coheight $n$ if $x \in V^{n}(H)$. For each integer $e>0$, the integer $\|e\| \geqslant 0$ is defined by

$$
p^{\|e\|} \leqslant e<p^{\|e\|+1} .
$$

A set of elements $x_{0}=1, x_{1}, \ldots, x_{n}$ ( $n$ finite) in $H$ is called an $n$-sequence of divided powers if

$$
\Delta\left(x_{i}\right)=\sum_{j=0}^{i} x_{j} \otimes x_{i-j}, \quad 0<i \leqslant n
$$

Theorem A [4, Lemma 7; AND 2, TheOREM 2]. Let $t<p^{n+1}$ and let $x_{0}$, $x_{1}, \ldots, x_{t-1}$ be a sequence of divided powers in $H$ where $x_{i}$ has coheight $n-\|i\|$, $0<i<t$. There is an element $x_{t}$ in $H$ of coheight $n-\|t\|$ such that $x_{0}$, $x_{1}, \ldots, x_{t-1}, x_{t}$ is a sequence of divided powers.

The following extension theorem of sequences of divided powers is a key lemma to determine the coalgebra structure of $H$ [4, Theorems 2 and 3].

The original proof of Sweedler, which consists of several steps, is done by induction on $n$ and $t$. In the following, we give an alternative proof, where we do not use induction, but the idea of relative Hopf modules [5] instead.

Received by the editors November 18, 1980.

1980 Mathematics Subject Classification. Primary 16A24.

Key words and phrases. Hopf algebra, sequence of divided powers.

${ }^{1}$ Supported in part by NSF Grant MCS-77-18723 A03.

(c) 1982 American Mathematical Society 0002-9939/82/0000-0404/\$02.00 
Proof. Replacing $H$ by $V^{n-\|t\|}(H)$, we may assume $n=\|t\|$. Let $\tilde{H}$ be the free $k$-algebra generated by $H$ and one indeterminate $z$. Thus, if $A$ is a $k$-algebra and $\varphi$ : $H \rightarrow A$ an algebra map, then for any $a \in A$, there is a unique algebra map $\tilde{\varphi}$ : $\tilde{H} \rightarrow A$ such that $\tilde{\varphi} \mid H=\varphi$ and $\tilde{\varphi}(z)=a$. Using this universal mapping property, define algebra maps

$$
\tilde{\Delta}: \tilde{H} \rightarrow \tilde{H} \otimes \tilde{H}, \quad \tilde{\varepsilon}: \tilde{H} \rightarrow k
$$

by the rule: $\tilde{\Delta} \mid H=\Delta$ (comultiplication of $H$ ), $\tilde{\Delta}(z)=z \otimes 1+1 \otimes z+\sum_{i=1}^{t-1} x_{i} \otimes$ $x_{t-i}, \tilde{\varepsilon} \mid H=\varepsilon$ (augmentation of $H$ ), $\tilde{\varepsilon}(z)=0$. Then $(\tilde{H}, \tilde{\Delta}, \tilde{\varepsilon})$ is an irreducible cocommutative Hopf algebra containing $H$ as a Hopf subalgebra. Since $x_{0}$, $x_{1}, \ldots, x_{t-1}, z$ is a $t$-sequence of divided powers in $\tilde{H}, V(z)=0$ if $p \nmid t$ and $V(z)=x_{s}$ if $t=p s$. In the latter case, $x_{s}$ has coheight $\|t\|-\|s\|=1$. Hence $V(z) \in V(H)$. Since $V$ is a semilinear Hopf algebra map, it follows that $V(\tilde{H})=$ $V(H)$. Let $\tilde{L}=P(\tilde{H})$, the primitives in $\tilde{H}$. Let $U$ (resp. $\tilde{U})$ be the restricted universal enveloping algebra of $L$ (resp. $\tilde{L})$. Then $U$ (resp. $\tilde{U}$ ) is a Hopf subalgebra of $H$ (resp. $\tilde{H})$ [3, Proposition 13.2.3]. We claim that the multiplication in $\tilde{H}$ induces an isomorphism

$$
H \otimes_{U} \tilde{U} \stackrel{\sim}{\rightarrow} \tilde{H} .
$$

Indeed, both sides are right $(\tilde{H}, \tilde{U})$-Hopf modules [5, p. 454] and the map is a homomorphism. Since $\tilde{H}$ is irreducible, $\tilde{H}$ is a free left (or right) $\tilde{U}$-module [5, Proposition 3]. Hence the category of right $(\tilde{H}, \tilde{U})$-Hopf modules is equivalent to the category of right $\tilde{H} / \tilde{H} \tilde{L}$-comodules [5, Theorem 1], where the equivalence is given by $M \mapsto M / M \tilde{L}$. If we apply this equivalence functor to the above homomorphism, we get the canonical map $H / H L \rightarrow \tilde{H} / \tilde{H} \tilde{L}$ which is an isomorphism, since $H / H L \simeq V(H), \tilde{H} / \tilde{H} \tilde{L} \simeq V(\tilde{H})$ and $V(\tilde{H})=V(H)$. This proves our claim.

Let $X$ be a basis of $\tilde{L}$ modulo $L$. Let $\Lambda$ be the set of all functions from $X$ to $\{0,1, \ldots, p-1\}$ with finite support. Give a total order on $X$. For each $f$ in $\Lambda$, put

$$
[f]=\frac{c_{1}^{e_{1}} \cdots c_{n}^{e_{n}}}{e_{1} ! \cdots e_{n} !} \text { and }|f|=e_{1}+\cdots+e_{n}
$$

where $\left\{c_{1}, \ldots, c_{n}\right\}$ is the support of $f$ with $c_{1}<\cdots<c_{n}$ and $e_{i}=f\left(c_{i}\right)$. Then $\{[f] \mid f \in \Lambda\}$ is a free basis of the left $U$-module $\tilde{U}$ (Poincaré-Birkhoff-Witt), hence of the left $H$-module $\tilde{H}$, and we have

$$
\Delta[f]=\sum_{f=g+h}[g] \otimes[h] .
$$

Write $z=\Sigma_{f \in \Lambda} z_{f}[f], z_{f} \in H$. Then

$$
\tilde{\Delta}(z)=\Sigma \Delta\left(z_{g+h}\right)([g] \otimes[h])
$$

where the sum is taken over the set of all $g, h \in \Lambda$ with $g+h \in \Lambda$. Since $\tilde{\Delta}(z)=z \otimes 1+1 \otimes z+\sum_{i=1}^{t-1} x_{i} \otimes x_{t-i}$, and $\{[g] \otimes[h] \mid g, h \in \Lambda\}$ is a free basis of the left $H \otimes H$-module $\tilde{H} \otimes \tilde{H}$, it follows from comparison of the coefficients that $z_{f}=0$ for $|f|>1$ and $z_{f} \in k$ for $|f|=1$. Put $x_{t}=z-\Sigma_{|f|-1} z_{f}[f]$. Then $x_{t} \in H$ and $\tilde{\Delta}(z)-z \otimes 1-1 \otimes z=\Delta\left(x_{t}\right)-x_{t} \otimes 1-1 \otimes x_{t}$. Hence $x_{0}$, $x_{1}, \ldots, x_{t-1}, x_{t}$ is a sequence of divided powers in $H$. Q.E.D. 
The above idea of proof yields more general results. Note that we merely used the fact that $V(H)=V(\tilde{H})$ in the latter part of the above proof. Hence, what we proved actually is the following

THEOREM B. Let $\tilde{\boldsymbol{H}}$ be an irreducible cocommutative Hopf algebra and let $\boldsymbol{H} \subset \tilde{H}$ be a Hopf subalgebra. Assume $V(H)=V(\tilde{H})$. If $z \in \tilde{H}$ satisfies

$$
\Delta(z)-z \otimes 1-1 \otimes z \in H \otimes H
$$

there is an element $x \in H$ such that

$$
\Delta(z)-z \otimes 1-1 \otimes z=\Delta(x)-x \otimes 1-1 \otimes x .
$$

It is enough to assume $V(z) \in V(H)$ instead of $V(H)=V(\tilde{H})$. (Replace $\tilde{H}$ by the Hopf subalgebra generated by $H$ and $z$.)

The above theorem can be interpreted as a cohomological vanishing theorem of the underlying coalgebras of irreducible cocommutative Hopf algebras. To clarify the meaning, for a pointed irreducible cocommutative coalgebra $C$, let $C^{+}=$ $\operatorname{Ker}(\varepsilon)$ and

$$
\delta: C^{+} \rightarrow C^{+} \otimes C^{+}, \quad \delta(x)=\Delta(x)-x \otimes 1-1 \otimes x
$$

where 1 denotes the unique group-like element of $C$. We want to determine the image $\delta\left(C^{+}\right)$. Let $\delta_{n}: C^{+} \rightarrow \otimes^{n+1} C^{+}$be the $n$ times iterated $\delta$-map. Let

$$
u=\sum_{i} x_{i} \otimes y_{i} \in C^{+} \otimes C^{+}
$$

be an element satisfying

(a) $\Sigma_{i} x_{i} \otimes y_{i}=\Sigma_{i} y_{i} \otimes x_{i}$

(b) $\Sigma_{i} \delta\left(x_{i}\right) \otimes y_{i}=\Sigma_{i} x_{i} \otimes \delta\left(y_{i}\right)$.

There is a pointed irreducible cocommutative coalgebra $C^{u}=C \oplus k z$ which contains $C$ as a subcoalgebra and satisfies

$$
\Delta(z)=z \otimes 1+1 \otimes z+u, \quad \varepsilon(z)=0 .
$$

Then $V(z)$ is determined by $u$ as follows: $\sum_{i} \delta_{p-2}\left(x_{i}\right) \otimes y_{i}$ is a symmetric tensor in $\otimes^{p} C^{+}$. Let

$$
v:\left(\text { the symmetric tensors in } \otimes^{p} C^{+}\right) \rightarrow k^{1 / p} \otimes C^{+}
$$

be the $\frac{1}{p}$-linear map defined [1, Theorem 4.1.1(a), p. 273] (where denoted by $V$ ). Put $v(u)=v\left(\sum_{i} \delta_{p-2}\left(x_{i}\right) \otimes y_{i}\right)$. Then $V(z)$ is precisely $v(u)$.

If $C$ underlies a Hopf algebra, then the image $\delta\left(C^{+}\right)$can be characterized as follows.

THEOREM C. Let $H$ be an irreducible cocommutative Hopf algebra. The image $\delta\left(H^{+}\right)$is precisely the set of elements $u$ in $H^{+} \otimes H^{+}$satisfying (a), (b) and (c) $v(u) \in V(H)$.

Proof. If $u=\delta(x)$ with $x \in H^{+}$, then $u$ satisfies (a), (b) and $v(u)=V(x) \in$ $V(H)$. Conversely, if $u$ satisfies (a), (b), (c), let $\tilde{H}$ be the Hopf algebra generated by $H$ and one indeterminate $z$ with $\delta(z)=u, \varepsilon(z)=0$. It follows from $V(z)=v(u) \in$ $V(H)$ that $V(H)=V(\tilde{H})$. Hence $\delta(z)=\delta(x)$ for some $x \in H$ by Theorem B. Q.E.D. 
Theorem A follows from Theorem $\mathrm{C}$ applied to $u=\sum_{i=1}^{t-1} x_{i} \otimes x_{t-i}$ and $V^{n-\|t\|}(H)$ as $H$.

\section{REFERENCES}

1. R. Heyneman and M. E. Sweedler, Affine Hopf algebras. II, J. Algebra 16(1970), 271-297.

2. K. Newman, Sequences of divided powers in irreducible, cocommutative Hopf algebras, Trans. Amer. Math. Soc. 163 (1972), 25-34.

3. M. E. Sweedler, Hopf algebras, Benjamin, New York, 1969.

4. __ Hopf algebras with one group-like element, Trans. Amer. Math. Soc. 127 (1967), 515-526.

5. M. Takeuchi, Relative Hopf modules, J. Algebra 60 (1979), 452-471.

School of Mathematics, The Institute for Advanced Study, Princeton, New Jersey 08540

Current address: Department of Mathematics, University of Tsukuba, Ibaraki 305, Japan 